\title{
Critical Retardation Effects and Slow Relaxations
}

\section{The problem of slow relaxations}

From the viewpoint of experimental workers, slow relaxations are abnormally (i.e. unexpected) slow transition processes. The time of a transition process is determined as that of the transition from the initial state to the limit $(t \rightarrow \infty)$ regime. The limit regime itself can be a steady state, a limit cycle (a self-oscillation process), a strange attractor (stochastic self-oscillation), etc.

Inevitably, the "limit" regime of the process is observed in experiments with finite accuracy. It is possible that the experiment was too short in time, and if the period of this experiment is sufficiently long, the regime would change considerably. Strictly speaking the limit transition $t \rightarrow \infty$ holds only for a mathematical model.

Similarly, the achievement of the limit regime (the end of the transition process) is only determined with finite accuracy. This circumstance will be used constantly in what follows.

The most difficult problem is the adequate determination of the "abnormally" (i.e. unexpected) slow transition process. For this purpose, we must imagine the simplest system of expectations. It is based on the hypotheses about reaction mechanisms. A catalytic reaction is represented as a combination of elementary steps (see Chap. 3). We admit some hypotheses concerning values of the corresponding rate coefficients. Typical concentrations of gas-phase substances and of surface compounds are also assumed to be known. One can also introduce a concept of the characteristic time of a step. For example, the characteristic time for the step $\mathrm{A} \rightleftarrows \mathrm{B}$ can be determined as $1 /\left(k^{+}+k^{-}\right)$, where $k^{+}$and $k^{-}$are the rate constants for the direct and reverse reactions. For the reaction $\mathrm{A}+\mathrm{B} \rightarrow \mathrm{C}$ one can introduce two characteristic times: $1 / k C_{\mathrm{A}}$ and $1 / k C_{\mathrm{B}}$, where $C_{\mathrm{A}}$ and $C_{\mathrm{B}}$ are the characteristic concentrations of $\mathrm{A}$ and $\mathrm{B}$.

Hence, as a rule, the research worker has some knowledge (some hypothesis) about the base steps and their characteristic times.

One refinement is necessary here. If a transition process is examined for the reactor as a whole, the equations of the steps include all the reacting substances. If the transition process is examined only on the surface, the equations of the steps contain only surface compounds, and the concentrations of gas-phase substances are included in the rate constants.

Let some transition process proceed much slower than might have been expected in terms of the hypotheses about characteristic times for the steps 
(slow relaxation). Every time slow relaxations are observed in experiments, there arises a question: what are the reasons for them? To answer this question in some specific cases, it is useful to have a list of probable reasons for slow relaxations of catalytic reactions.

Difficulties of the interpretation of experimental data on the kinetics of heterogeneous catalytic reactions are conditioned by the following.

(1) One can observe only an insignificant part of substantial variables (difficulties in the experimental determination of concentrations for intermediates during the reaction process are well known).

(2) In addition to kinetic processes, the contribution of some "side" processes can also be essential. Among these processes there are the diffusion of reactants into the catalyst bulk and any effect of the reaction media on catalysts (catalytic corrosion, reconstruction of a catalyst surface layer caused by reaction, etc.).

Side processes often proceed more slowly than kinetic reactions, from which a first explanation follows: slow relaxations are induced by the effect of side processes $[1,2]$.

Another variant of the explanation is: the list of substances and the reaction mechanism is incomplete and so slow relaxations are explained by the slow steps that have not been taken into account. It must be emphasized that slow transition processes can also be caused by slow steps, in those cases in which the steady state rate of a catalytic reaction is high. This can be exemplified by two linear catalytic cycles connected by a slow step

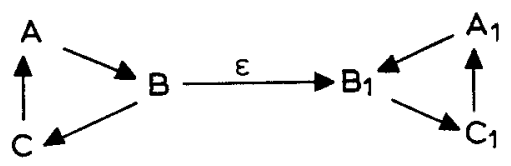

In this case the steady state rate is controlled by the rate constants of the $A_{1} \rightarrow B_{1} \rightarrow C_{1} \rightarrow A_{1}$ cycle, whereas the transition process is limited by the slow reaction connecting cycles.

Finally, we can suggest a third explanation: fast steps can compose a mechanism with slow relaxations. Indeed, nothing suggests that the relaxation time for a set of chemical kinetic equations is directly dependent on the characteristic times of the individual steps. But it cannot be treated as a reason for slow relaxations. It is only a simple indication for the possibility of finding such reasons here. Let us now indicate the reasons according to which fast steps can compose a mechanism with slow relaxations.

First, there must be a large number of reacting substances. Even for linear reaction mechanisms, there does not exist a simple "rule of adding" characteristic times for the steps forming a reaction mechanism. For example, let us consider a linear irreversible cycle $A_{1} \rightarrow A_{2} \rightarrow \ldots \rightarrow A_{n} \rightarrow A_{1}$ in which all the constants are the same and equal to $k$. The vector of concentrations 
$\vec{c}$ can be described by the equation $\mathrm{d} \vec{c} / \mathrm{d} t=\vec{K} \vec{c}$. The matrix $\vec{K}$ has the eigenvalues

$$
\begin{aligned}
\lambda_{l} & =k\left(1^{1 / n}-1\right) \\
& =k \exp \left\{i \frac{2 \pi l}{n}-1\right\} \quad l=1, \ldots, n
\end{aligned}
$$

The rate of relaxation is determined by the non-zero value the $\{\operatorname{Re} \lambda \mid$ closest to zero, which is equal to.

$$
k\left(1-\cos \frac{2 \pi}{n}\right) \approx 2 k\left(\frac{\pi}{n}\right)^{2}
$$

at high $n$. The corresponding characteristic time $\tau=1 /|\operatorname{Re} \lambda|$ increases with increasing $n$ as $n^{2}$.

Secondly, in some cases the presence of high rate constants leads to slow transition processes. The simplest example is

$$
\mathrm{C} \underset{k_{2}}{\stackrel{k_{2} 3}{\rightleftarrows}} \mathrm{A} \stackrel{k_{1}}{\longrightarrow} \mathrm{B}
$$

Non-zero eigenvalues are determined explicitly as

$$
\lambda_{1,2}=-\frac{k_{1}+k_{2}+k_{3}}{2} \pm \frac{1}{2}\left[\left(k_{1}+k_{2}+k_{3}\right)-4 k_{1} k_{3}\right]^{1 / 2}
$$

Let the constant $k_{2}$ be very high: $k_{2} \gg k_{1}, k_{3}$. Then we obtain

$$
\lambda_{1,2} \approx-\frac{k_{1}+k_{2}+k_{3}}{2} \pm\left[\frac{k_{1}+k_{2}+k_{3}}{2}-\frac{k_{1} k_{3}}{k_{1}+k_{2}+k_{3}}\right]
$$

In this case the eigenvalue closest to zero and the respective relaxation times will be

$$
\begin{aligned}
\lambda & \approx \frac{k_{1} k_{3}}{k_{1}+k_{2}+k_{3}} \\
\tau & \approx \frac{k_{1}+k_{2}+k_{3}}{k_{1} k_{3}}
\end{aligned}
$$

If $k_{2} \rightarrow \infty$ ( $k_{1}$ and $k_{3}$ being restricted), we will obtain $\lambda \rightarrow 0, \tau \rightarrow \infty$.

Finally, let us discuss the most important reason, from our point of view, that, in particular, causes slow relaxations in $\mathrm{CO}$ oxidation over $\mathrm{Pt}$.

If a system of chemical kinetic equations is non-linear and the reaction mechanism includes an interaction step between various substances, bifurcations are possible. They account for the effects of critical retardation. Let us illustrate this by the simplest (non-chemical) example. Consider the differential equation

$$
\dot{x}=-\alpha x-x^{3}
$$


For $\alpha \geqslant 0$ it has only one fixed point at $x=0$. For $\alpha \geqslant 0$ this point is stable, but for $\alpha<0$ the equation will have three fixed points $\left[x=0, \pm\left(-\alpha^{1 / 2}\right)\right]$ and the point $x=0$ is unstable. In the cases $\alpha>0$ and $\alpha \rightarrow 0$, the relaxation of the system towards the point $x=0$ becomes still slower. The solution tends to zero as $\exp (-\alpha t)$ [more precisely $x(t)=\exp (-\alpha t)\left(\alpha A / 1-A \mathrm{e}^{-2 \alpha t}\right)^{1 / 2}$ where $\left.A=x_{0}^{2} /\left(x_{0}+x_{0}^{2}\right), x_{0}=x(0)\right]$ tends to zero. At a point of bifurcation $(\alpha=0)$, the equation reduces to $x=-x^{3}$, and the solution tends to zero more slowly than any exponent $x(t)=1 /[2(t-c)]^{1 / 2}$ where $c$ is a constant.

In the case of steady state bifurcations, certain eigenvalues of the linearapproximation matrix reduce to zero. If we consider relaxations towards a steady state, then near the bifurcation point their rates are slower. This holds for the linear approximation in the near neighbourhood of the steady state. Similar considerations are also valid for limit cycles. But is it correct to consider the relaxation of non-linear systems in terms of the linear approximations? To be more precise, it is necessary to ask a question as to whether this consideration is sufficient to get to the point. Unfortunately, it is not since local problems (and it is these problems that can be solved in terms of the linear approximations) are more simple than global problems and, in real systems, the trajectories of interest are not always localized in the close neighbourhood of their attractors.

Here we face a strange situation. Until recently there was no theory of transition processes in dynamic systems. We observe a sharp contrast with the theory of limit behaviour that was the subject of a large number of investigations [3-12]. If some data concerning transition processes were reported, they were largely a secondary product of studying the limits $t \rightarrow \infty$.

To interpret the problem under discussion concerning slow relaxations in chemistry, it was necessary to clarify what must be regarded as slow relaxations of dynamic systems (i.e. to introduce some reasonable definition). In addition, it was necessary to find connections of slow relaxations with bifurcations and other dynamic peculiarities. This has been done by Gorban' et al. [13-19].

In this chapter we will suggest a theory of transition processes and slow relaxations in dynamic systems. The inclusion of such mathematical sections to a book on chemical kinetics is dictated by the necessity to understand the details of slow transition processes in the absence of a comprehensive and clear representation of the theory of slow relaxations.

\section{The limit behaviour of dynamic systems}

Here we must make a great jump into some generalities of our presentation. To discuss reasons for the slow transition processes in non-linear systems, we do not need the formalism of chemical kinetics. To begin with we need very little: a concept about the phase space $\vec{X}$ and the time shift $\vec{T}_{t}$, 
that is the transforming " $\vec{X}$ now" into " $\vec{X}$ after the time $t$ ". So far we do not even need differential equations to describe the system. We shall use directly their solutions specified by the $T$ mapping.

For the kinetic equations $\vec{c}=\mathrm{f}(\vec{c})$ let us consider a reaction polyhedron $\vec{D}$ in the phase space $\vec{X}$. For the point $\vec{x} \in \vec{D}$ and for $t>0$ the value of $\vec{T}_{t}(\vec{x})$ will be determined as $\vec{T}_{t}(\vec{x})=\vec{c}(t, \vec{x})$, where $\vec{c}(t, \vec{x})$ is the solution of the equations $\vec{c}=\mathrm{f}(\vec{c})$ with the initial conditions $\vec{c}(0)=\vec{x}$.

The $\vec{T}$ mapping is not given analytically from the beginning. It is determined with the help of the solution of a system of differential equations. In this sense we do not have it, but we can specify and research general properties of $\vec{T}$.

The language applied is poorer and hence is more simple than that of the theory of differential equations. It is the language of topological dynamics [3-7]. Let us introduce the main concepts required here and in what follows.

$\vec{X}$ is the compact metric space (to be more definite, it is a closed restricted subset of $\left.\vec{R}^{n}\right)$. Let $\rho(\vec{x}, \vec{y})$ be the distance between the points $\vec{x}$ and $\vec{y}$. For $t>0, \bar{T}_{t}$ is the continuous one-to-one mapping of $\vec{X}$ to $\bar{X}$ (time shift) and $\vec{T}_{0}=$ id (identity mapping): $\vec{T}_{0}(\vec{x})=\vec{x}$.

$$
\vec{T}_{t} \vec{T}_{t^{\prime}}=\vec{T}_{t+t^{\prime}}
$$

The equality (1) means that, after shifting the system for $t$ and then for $t^{\prime}$, we will obtain a system shifted for $t+t^{\prime}$. The function $\vec{T}_{t}(x)$ is continuous relative to the totality of arguments $t$ and $x$.

For $\vec{x} \in \vec{X}$, the function that puts each $t \in[0, \infty]$ in correspondence with the point $\vec{T}_{t}(\vec{x})$ is called $\vec{x}$-motion. For certain $\vec{x} \in \vec{X}$ this function can also be extended to the negative value of $t$, i.e. if for certain $\vec{y} \in \vec{X}$ and $t \geqslant 0$ the condition $\vec{T}_{t}(\vec{y})=\vec{x}$ holds, then we will assume that $\vec{T}_{-t}(\vec{x})=\vec{y}$. In what follows we will use this extension without additional explanation. To avoid misunderstanding, it must be remembered that $\vec{T}_{-t}$ is not determined throughout the whole $\vec{X}$ (in contrast to $\vec{T}_{l}, t>0$ ). $\vec{x}$-Motion will be called a whole if $\vec{T}_{t}(\vec{x}) \in \vec{X}$ is determined throughout the whole time axis $t \in(-\infty, \infty)$.

A positive $\vec{x}$-semitrajectory is defined as the set (a curve) $\left\{\vec{T}_{t}(\vec{x}) / t \in[0, \infty)\right\}$. If the $\vec{x}$-motion is a whole, then the negative semitrajectory $\left\{\vec{T}_{t}(\vec{x}) / t \in(-\infty\right.$, $0]\}$ has also been determined. The union of positive and negative semitrajectories is called a whole trajectory.

The point $\vec{y} \in \vec{X}$ is called a $\omega$-limit for the $\vec{x}$-motion if there exists such a sequence $t_{i} \rightarrow \infty$ that

$$
\vec{T}_{-t_{i}}(\vec{x}) \rightarrow \vec{y}
$$

A set of the $\omega$-limit points of the $\vec{x}$-motion is called a $\omega$-limit set. We will denote it as $\omega(\vec{x})$. In terms of the suggested compactness of $\vec{X}$ and continuity of $\vec{T}$, it follows that, for any $\vec{x} \in \vec{X}$, the $\omega$-limit set $\omega(\vec{x})$ is non-void.

If the $\vec{x}$-motion is whole, then we define $\alpha$-limit points for it. The point $\vec{y} \in \vec{X}$ is called an $\alpha$-limit point for the whole $\vec{x}$-motion if there exists such a sequence of $t_{i} \rightarrow \infty$ that 


$$
\overleftrightarrow{T}_{-t_{i}}(\vec{x}) \rightarrow \vec{y}
$$

A set of $\alpha$-limit points for the whole $\vec{x}$-motion will be denoted as $\alpha(\vec{x})$. For the whole $\vec{x}$-motions, $\alpha(\vec{x})$ will be non-void.

If $\vec{x} \in \omega(\vec{x})$, the $\vec{x}$-motion is called $\mathrm{P}^{+}$-stable (positively Poisson-stable).

If the $\vec{x}$-motion is whole, not $\mathrm{P}^{+}$-stable $[\vec{x} \notin \omega(\vec{x})]$ and $\alpha(\vec{x}) \bigcap \omega(\vec{x}) \neq \phi$, then the $\vec{x}$-trajectory will be called a loop. An example of a loop on the plane is a loop of the sepatrix that is a trajectory going from a singular point and back to it. Another example is a homoclinic trajectory for which the same saddle limit cycle is both a $\alpha$-limit and an $\omega$-limit set.

It is possible to introduce an $\omega$-limit set of the whole system as a union of $\omega$-limit sets for individual points

$$
\omega_{T}=\bigcup_{\vec{x} \in \vec{X}} \omega(\vec{x})
$$

In what follows we will often consider systems that are parametrically dependent (physical parameters will mean temperature, pressure, inlet concentrations of reactants, etc.). A vector of the parameters will be denoted by $\vec{k}$ and the set $\vec{K}$ is assumed to be compact (a closed bounded subset $\vec{R}^{n}$ ).

For every $\vec{k}$ there exists a definite mapping $\vec{T}$. Let us denote its value as $\vec{T}_{t}(\vec{x}, \vec{k})$. The function $\vec{T}_{t}(\vec{x}, \vec{k})$ is assumed to be continuous in the union of arguments. The $\vec{x}$-motion for a given $\vec{k}$ will be termed an $(\vec{x}, \vec{k})$-motion. The $\omega$-limit set of the $(\vec{x}, \vec{k})$-motion will be denoted as $\omega(\vec{x}, \vec{k})$. If the $(\vec{x}, \vec{k})$-motion is whole, its $\alpha$-limit set will be denoted as $\alpha(\vec{x}, \vec{k})$. At a given $\vec{k}$, the $\omega$-limit set for the whole of system (4) is denoted as $\omega_{T}(\vec{k})$.

The $\omega$-limit set is a very natural object from the qualitative viewpoint. $\omega(\vec{x}, \vec{k})$ is the set towards which the $\vec{x}$-motion tends for $t \rightarrow \infty$ (for a given $\vec{k}$ ). Therefore it seems a natural formalization of the concept of a "limit regime". The $\omega$-limit set can consist of one fixed point (steady-state) and points belonging to one limit cycle. But its structure can also be more complex, i.e. it can include several fixed points and cycles, various surfaces, strange attractors, etc.

\section{Relaxation times. Determination of slow relaxations}

System relaxation can be represented as a motion from the initial state $\vec{x}$ towards the $\omega$-limit set $\omega(\vec{x}, \vec{k})$. Relaxation time can be defined as the time of this motion. But here we have several possibilities.

Let us take some $\varepsilon>0$ as the accuracy for the achievement of a limit set. $\tau_{1}(\vec{x}, \vec{k}, \varepsilon)$ will be the time for the first entry of the system from its initial state into the $\varepsilon$-neighbourhood of $\omega(\vec{x}, \vec{k})$ (for a given $\vec{k})$. After then the system can leave the $\varepsilon$-neighbourhood and then enter it again and do this several (even many) times. Finally, the $(\vec{x}, k)$-motion will constantly remain in this neighbourhood, but this process can take more time than it needed for the first 
entry. Therefore let us introduce the time for the residence of $(\vec{x}, \vec{k})$-motion outside the $\varepsilon$-neighbourhood of $\omega(\vec{x}, \vec{k})\left[\tau_{2}(\vec{x}, \vec{k}, \varepsilon)\right]$ and the time for its final entry into this neighbourhood $\left[t_{3}(\vec{x}, \vec{k}, \varepsilon)\right]$. Hence

$$
\begin{aligned}
& \tau_{1}(\vec{x}, \vec{k}, \varepsilon)=\inf \left\{t>0 / \rho\left[\vec{T}_{t}(\vec{x}, \vec{k}), \omega(\vec{x}, \vec{k})\right]<\varepsilon\right\} \\
& \tau_{2}(\vec{x}, \vec{k}, \varepsilon)=\operatorname{mes}\left\{t>0 / \rho\left[\vec{T}_{t}(\vec{x}, \vec{k}), \omega(\vec{x}, \vec{k})\right] \geqslant \varepsilon\right\} \\
& \tau_{3}(\vec{x}, \vec{k}, \varepsilon)=\inf \left\{t>0 / \rho\left[\vec{T}_{t}(\vec{x}, \vec{k}), \omega(\vec{x}, \vec{k})\right]<\varepsilon, \text { if } t^{\prime}>t\right\}
\end{aligned}
$$

where mes is the Lebesgue measure (on a straight line it is length) and $\rho$ is the distance in $\vec{X}$ [the distance from the point $(\vec{y})$ towards the set $(\vec{P})$ is determined as $\left.\rho(\vec{y}, \vec{P})=\inf _{\vec{a} \in \vec{P}} \rho(\vec{y}, \vec{z})\right]$.

At the end of Sect. 2, we agreed that the relaxation of $(\vec{x}, \vec{k})$-motion is the tendency to $\omega(\vec{x}, \vec{k})$ that is its $\omega$-limit set. But in this case another viewpoint is possible. We can treat $\omega_{T}(\vec{k})$ as a set of limit regimes and define relaxation as a motion towards it. Then it is not important which of the $\omega$-limit points the $(\vec{x}, \vec{k})$-motion has been approached, either its own [from $\omega(\vec{x}, \vec{k})$ ] or foreign [from $\omega(\vec{y}, \vec{k})$ for $\vec{y} \neq \vec{x}$ ]. Of importance is the approach to the complete set of limit regimes $\omega_{T}(\vec{k})$. In this connection we introduce three relaxation times [analogous to $\tau_{1,2,3}$ of eqns. (5)].

$$
\begin{aligned}
& \eta_{1}(\vec{x}, \vec{k}, \varepsilon)=\inf \left\{t>0 / \rho\left[\vec{T}_{t}(\vec{x}, \vec{k}), \omega_{T}(\vec{k})\right]<\varepsilon\right\} \\
& \eta_{2}(\vec{x}, \vec{k}, \varepsilon)=\operatorname{mes}\left\{t>0 / \rho\left[\vec{T}_{t^{\prime}} \cdot(\vec{x}, \vec{k}), \omega_{T}(\vec{k})\right] \geqslant \varepsilon\right\} \\
& \eta_{3}(\vec{x}, \vec{k}, \varepsilon)=\inf \left\{t>0 / \rho\left[\vec{T}_{t^{\prime}}(\vec{x}, \vec{k}), \omega_{T}(\vec{k})\right]>\varepsilon, \text { if } t^{\prime}>t\right\}
\end{aligned}
$$

Let us now define what must be considered as a slow relaxation process. To judge whether the relaxation time is large or small, it must be compared with some specified time scale. But the system has no such specified time scale. Besides, with decreasing $\varepsilon$, relaxation times can become arbitrarily large even for the motion towards the only stable fixed point.

For each initial state $\vec{x}$ and $\vec{k}, \varepsilon$ values all relaxation times are finite. But their set can be unrestricted for $\vec{k} \in \vec{K}, \vec{x} \in \bar{X}$ (but for fixed $\varepsilon>0$ ). It is in this case that we will speak about slow relaxations.

As the simplest example, let us consider the differential equation $\dot{x}=x^{2}-1$ on the segment $X=[-1,1]$. The point $x=-1$ is stable and the point $x=1$ is unstable. For any fixed $\varepsilon>0, \varepsilon<1 / 2$ the relaxation times $\tau_{1,2,3}, \eta_{3} \rightarrow \infty$ at $x \rightarrow 1, x<1$, since $x$-motion delays near the fixed point. In this system, the times $\eta_{1}$ and $\eta_{2}$ are restricted for any $\varepsilon>0$.

Definition 1. We will suggest that the system has $\tau_{i}-\left(\eta_{i}\right)$ slow relaxations if, for some $\varepsilon>0$, the function $\tau_{i}(\vec{x}, \vec{k}, \varepsilon)\left[\eta_{i}(\vec{x}, \vec{k}, \varepsilon)\right]$ is not restricted from above in $\vec{X} \times \vec{K}$, i.e. for any $t>0$ there could be found such $\vec{x} \in \dot{X}, \vec{k} \in \vec{K}$ that $\tau_{i}(\vec{x}, \vec{k}, \varepsilon)>t\left[\eta_{i}(\vec{x}, \vec{k}, \varepsilon)>t\right]$.

Relationships between various types of slow relaxations are determined by the inequalities $\tau_{i} \geqslant \eta_{i}, \tau_{1} \leqslant \tau_{2} \leqslant \tau_{3}, \eta_{1} \leqslant \eta_{2} \leqslant \eta_{3}$. If there are $\eta_{1}$ slow relaxations, all the others exist. Some examples can be given for the exist- 
ence of $\eta_{2}$ slow relaxations without $\eta_{1}$ slow relaxations. Directly from the definitions and the compactness of $\vec{X}$ it follows that, in a separate system $(\vec{k}$ is fixed) $\eta_{1}$ slow relaxations are impossible. But for $\eta_{2}$ slow relaxations this is not valid.

Example 1. The existence of $\eta_{2}$ slow relaxations in the system that is independent of the parameter. Let us consider a system on the plane in the circle $x^{2}+y^{2} \leqslant 1$, specified in the polar coordinates by the equations

$$
\begin{aligned}
& \dot{r}=-r(1-r)(r \cos \phi+1) \\
& \dot{\phi}=r \cos \phi+1
\end{aligned}
$$

The complete $\omega$-limit set consists of two fixed points $r=0$ and $r=1, \phi=\pi$ [Fig. 1(a)]. The boundary of the circle consists of a fixed point $r=1, \phi=\pi$ and a loop going from this fixed point and returning to it. If the initial point of the motion $\left(r_{0}, \phi_{0}\right)$ lies near the boundary inside the circle $\left(r_{0}<1\right)$, the motion is delayed for a long time near the boundary. In this case it periodic-

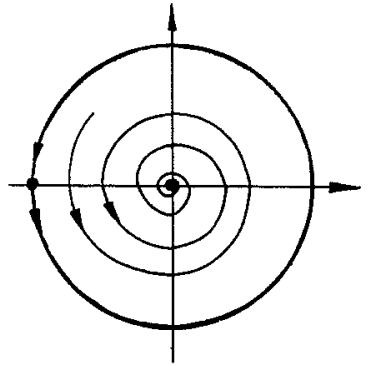

(a)

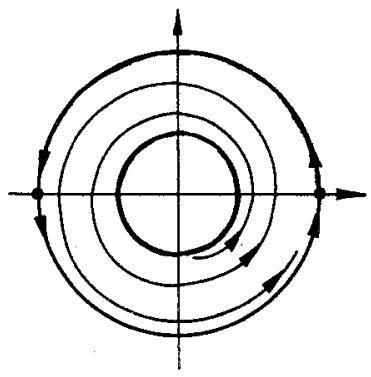

(c)

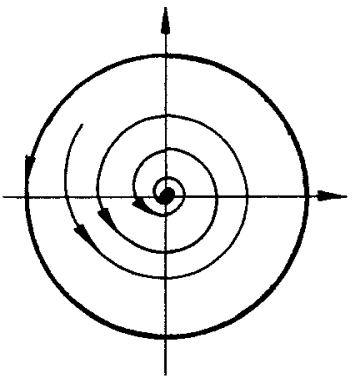

(b)

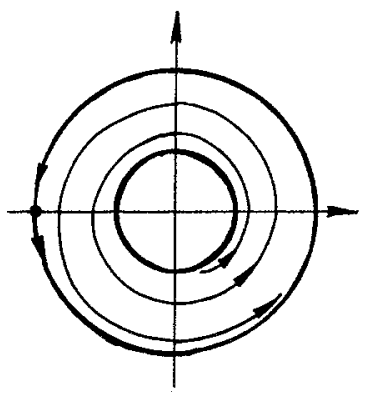

(d)

Fig. 1. Phase patterns of (a) system (7); (b) system 8; (c) system (9); (d) system (10). 
ally enters the close neighbourhood of the $\omega$-limit point $r=1, \phi=\pi$, so that $\eta_{1}$ is finite for $r_{0} \rightarrow 1$. But the residence time $\eta_{2}$ outside the close neighbourhood of this point tends to $\infty$ for $r_{0} \rightarrow 1$.

Several simple examples given below will illustrate the existence of one type for slow relaxations without others. We will not go into detail to explain why these examples deal with one or another type of slow relaxation. It can be seen from the equations and phase patterns (Fig. 1).

Example 2. There exist $\eta_{3}$ but no $\eta_{2}$ slow relaxations. In the above example let us replace the boundary loop by an unstable limit cycle

$$
\begin{array}{r}
\dot{r}=-r(1-r) \\
\phi=1
\end{array}
$$

Now the complete $\omega$-limit set $\omega_{T}$ includes the overall boundary circle and the point $r=0$ [Fig. 1(b)]. Residence time outside the $\varepsilon$ neighbourhood of $\omega_{T}$ is restricted for any $\varepsilon>0$. But $\eta_{3}\left[\left(r_{0}, \phi_{0}\right), 1 / 2\right] \rightarrow \infty$, if $r_{0} \rightarrow 1, r_{0} \neq 1$.

Example 3 . There exist $\tau_{1}$ but no $\eta_{2,3}$ slow relaxations. Let us consider the system in the ring $1 / 2 \leqslant x^{2}+y^{2} \leqslant 1$.

$$
\begin{aligned}
& \dot{r}=(1-r)(r \cos \phi+1)(1-r \cos \phi) \\
& \dot{\phi}=(r \cos \phi+1)(1-r \cos \phi)
\end{aligned}
$$

Here the complete $\omega$-limit set $\omega_{T}$ is the complete circle $r=1$ [Fig. 1(c)] For $r_{0}=1, \phi_{0} \rightarrow \pi, \phi_{0}>\pi$, the relaxation time $\tau_{1}\left(r_{0}, \phi_{0}, 1 / 2\right) \rightarrow \infty$, since for these initial points $\omega\left(r_{0}, \phi_{0}\right)=\{(r=1, \phi=0)\}$.

Example 4. There exist $\tau_{3}$ but no $\tau_{1,2}$ and no $\eta_{3}$ slow relaxations. Let us modify example 3 in the ring. We will take only one fixed point on the boundary circle $r=1$ [Fig. 1(d)]

$$
\begin{aligned}
& \dot{r}=(1-r)(r \cos \phi+1) \\
& \dot{\phi}=r \cos \phi+1
\end{aligned}
$$

In this case for $r_{0}=1, \phi_{0} \rightarrow \pi, \phi_{0}>\pi$, the relaxation time $\tau_{3}\left(r_{0}, \phi_{0}, 1 /\right.$ $2) \rightarrow \infty$. Relaxation times $\tau_{1,2}$ remain restricted, since now the motion delays near its $\omega$-limit set (in contrast to example 3 ).

Example 5. There exists $\tau_{2}$, but no $\tau_{1}$ and no $\eta_{2}$ slow relaxations. Here we will give an example for the system on a compact set that is not a variety (since $\bar{X}$ is a compact set, we will use this generality to simplify our problem). We will first consider a system in the ring $x^{2}+y^{2} \leqslant 2$, determined by the equations

$$
\begin{aligned}
& \dot{r}=-r(1-r)^{2}\left[(r \cos \phi+1)^{2}+r^{2} \sin \phi\right] \\
& \dot{\phi}=(r \cos \phi+1)^{2}+r^{2} \sin ^{2} \phi
\end{aligned}
$$




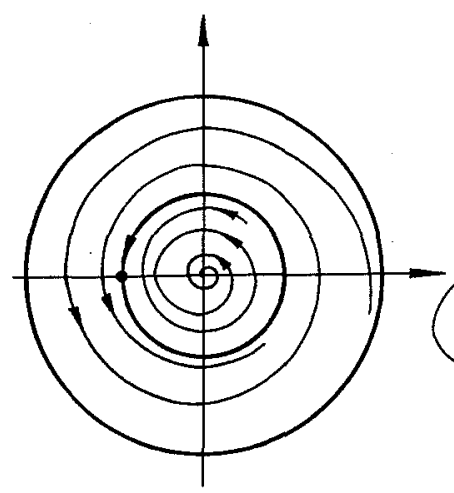

(a)

\section{(b)}

Fig. 2. Phase pattern of system (11)(a) without identification (pasting together) of fixed points; (b) after identification.

For this system the $\omega$-limit sets are [Fig. 2(a)]

$$
\omega\left(r_{0}, \phi_{0}\right)=\left\{\begin{array}{l}
\text { circumference } r=1, \text { at } r_{0}>1 \\
\text { point }(r=1, \phi=\pi), \text { at } r_{0}=1 \\
\text { point }(r=0), \text { at } r_{0}<1
\end{array}\right.
$$

Let us identify the fixed points $(r=1, \phi=\pi)$ and $(r=0)$ [Fig. 2(b)]. We will see that for $r_{0} \rightarrow 1, r_{0}<1$, the relaxation time $\tau_{2}\left(r_{0}, \phi_{0}, 1 / 2\right) \rightarrow \infty$, though $\tau_{1}$ and $\eta_{2}$ are restricted. In this case the time $\tau_{3}$ is unrestricted.

The majority of the above examples are non-rough (structurally unstable) systems. The rough dynamic systems on the plane cannot demonstrate the properties shown by the above examples. If $\vec{T}_{t}$ is specified by a rough individual (without parameters) system on the plane, there cannot exist $\eta_{1}, \eta_{2}$ slow relaxations and $\tau_{1,2,3}$ and $\eta_{3}$ slow relaxations can take place only simultaneously. This can be confirmed by the results given below and the data of some classical studies concerning smooth rough two-dimensional systems $[20,21]$.

\section{Bifurcations (explosions) of limit sets}

Here we treat bifurcations as peculiarities in the dependences $\omega(\vec{x}, \vec{k})$ on the initial $\vec{x}$ and the parameters $\vec{k}$ and of $\omega_{T}(\vec{k})$ on the parameters $\vec{k}$. This is a somewhat original viewpoint since, as usual, the dependence of the limit set on the initial data is not considered when studying bifurcations and attention is concentrated on its dependence on the parameters. The necessity to consider peculiarities of the $\omega(\vec{x}, \vec{k})$ function is due to our aim of examining slow relaxations.

The same aim also dictates the overall variety of peculiarities. Among the 
overall variety of continuity violations for $\omega(\vec{x}, \vec{k}), \omega_{T}(\vec{k})$ we have selected only two: the appearance of new limit points and of new limit sets.

The appearance of new limit points consists of the following. Let there exist a sequence of such $\vec{x}_{i} \rightarrow \vec{x}^{*}, \vec{k}_{i} \rightarrow \vec{k}^{*}$ for which $\omega\left(\vec{x}^{*}, \vec{k}^{*}\right)$ has such a point $\vec{y}$ that we obtain

$$
\rho\left[\vec{y}, \omega\left(\vec{x}_{i}, \vec{k}_{i}\right)\right]>\delta
$$

for a certain $\delta>0$ and every value of $i$. In this case we can say that $\omega(\vec{x}$, $\vec{k})$-bifurcations take place. The point $\left(\vec{x}^{*}, \vec{k}^{*}\right)$ will be called a point of the $\omega(\vec{x}$, $\vec{k}$ )-bifurcation. In a similar way, if $\vec{k}_{i} \rightarrow \vec{k}^{*}$ but there exists such $\vec{y} \in \omega_{T}\left(\vec{k}^{*}\right)$ that

$$
\rho\left[\vec{y}, \omega_{T}\left(\vec{k}_{i}\right)\right]>\delta
$$

for a certain $\delta>0$ and every value of $i$, we can say that the $\omega(\bar{k})$-bifurcation takes place. The point $\vec{k}^{*}$ will be called a point of the $\omega(\vec{k})$-bifurcation.

For the $\omega(\vec{x}, \vec{k})$ and $\omega(\vec{k})$-bifurcations, the following fact will be essential. In the limit set corresponding to the bifurcation point $\left(\vec{x}^{*}, \vec{k}^{*}\right)$ (or $\left.\vec{k}^{*}\right)$ there exists such a point $y$ which is localized at a finite distance from the whole of the sets $\left.\omega\left(\vec{x}_{i}\right), \vec{k}_{i}\right)$ [or $\left.\omega\left(\vec{k}_{i}\right)\right]$ for a certain sequence $\left(\vec{x}_{i}, \vec{k}_{i}\right) \rightarrow\left(\vec{x}^{*}, \vec{k}^{*}\right)$ [or $\left.\left(\vec{k}_{i} \rightarrow \vec{k}^{\star}\right)\right]$. The appearance of new $\omega$-limit sets consists in the following. Let there exist such sequences $\vec{x}_{i} \rightarrow \vec{x}^{*}, \vec{k}_{i} \rightarrow \vec{k}^{*}$ that for every $\vec{y} \in \omega\left(\vec{x}^{*}, \vec{k}^{*}\right)$

$$
\rho\left[\vec{y}, \omega\left(\vec{x}_{i}, \vec{k}_{i}\right)\right]>\delta
$$

for a certain $\delta>0$ and every value of $i$. In this case we say that there exist $\Omega(\vec{x}, \vec{k})$-bifurcations. In a similar way, if there are such sequences $\vec{k}_{i} \rightarrow \vec{k}^{*}$ and a point $\vec{x}^{*} \in \vec{X}$ that for every $\vec{y} \in \omega\left(\vec{x}^{*}, \vec{k}^{*}\right)$ we obtain

$$
\rho\left[\vec{y}, \omega\left(\vec{k}_{i}\right)\right]>\delta
$$

for a certain $\delta>0$ and every $i$, we can say the $\Omega(\bar{k})$-bifurcations take place.

$\Omega$-bifurcations are different for $\omega$-bifurcations. For the former at a finite distance from those limit sets that correspond to the converging sequences $\left[\left(\vec{x}_{i}, \vec{k}_{i}\right)\right.$ or $\left.\vec{k}_{i}\right]$ we can find all the points of a certain limit set $\omega\left(\vec{x}^{*}, \vec{k}^{*}\right)$ and not only some points of $\omega\left(\vec{x}^{*}, \vec{k}^{*}\right)\left[\right.$ or $\left.\omega\left(\vec{k}^{*}\right)\right]$.

The fact that $\omega$-bifurcations are possible without $\Omega$-bifurcations is not evident, and we therefore give the following example.

We will consider the example in which $\omega(\vec{x}, \vec{k})$-bifurcations exist but there are no $\Omega(\vec{x}, \vec{k})$-bifurcations. Let us first consider the system in the core (from $\left.\vec{R}^{3}\right) x^{2}+y^{2} \leq z^{2} ; 0 \leq z \leq 1$. Let it be set by the equations (in the cylindric coordinates: $x=r \cos \phi, y=r \sin \phi$ )

$$
\begin{aligned}
& \dot{r}=r(2 z-r-1)^{2}-2 r(1-r)(1-z) \\
& \dot{\phi}=r \cos \phi+1 \\
& \dot{z}=-z(1-z)^{2}
\end{aligned}
$$




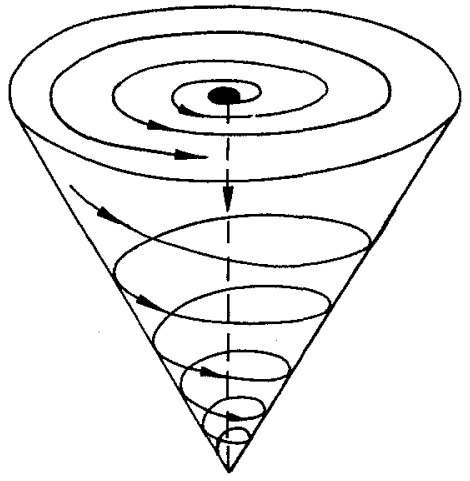

(a)

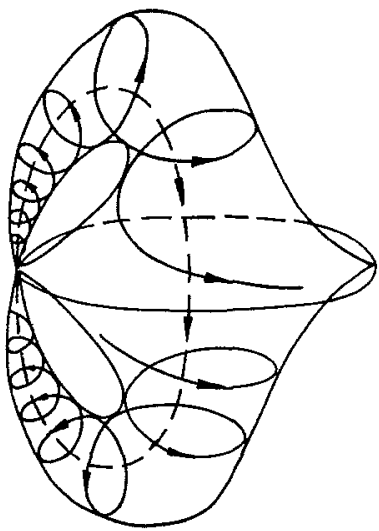

(b)

Fig. 3. $\omega(\vec{x}, \vec{k})$. but no $\Omega(\vec{x}, \vec{k})$-bifurcation. (a) Phase pattern of system (14); (b) as (a) after identification of all the fixed points.

For $t \rightarrow \infty$ the solutions of eqns. (14) behave as follows (depending on the initial conditions). If $0 \leq z(0) \leq 1,0<r(0) \leq z(0)$, then for $t \rightarrow \infty$ the solution tends to its unique $\omega$-limit point, which is a fixed point $z=r=0$. If $z(0)=1,0<r(0)<1$, then for $t \rightarrow \infty$ the solution is "winding" inside around the circle $z=r=1$. If the initial point lines on this circle $[z(0)=1$, $r(0)=1]$, then the $\omega$-limit point is unique: $z=1, r=1, \phi=\pi$. In case $z(0)=1, r(0)=0$, the $\omega$-limit point is also unique: $z=1, r=0$. The phase pattern is represented in Fig. 3(a).

Let us consider a sequence of the cone points $\left(r_{n}, \phi_{n}, z_{n}\right) \rightarrow\left(r^{*}, \phi^{*}, 1\right)$, $r^{\star} \neq 0,1, z_{n}<1$ for every value of $n$. For every point of the sequence, the $\omega$-limit set consists of an unique point and for $\left(r^{*}, \phi^{*}, 1\right)$ of a circle. If we identify all the equilibrium state [Fig. 3(b)], we will have $\omega(\vec{x}, \vec{k})$-bifurcations but no $\Omega(\vec{x}, \vec{k})$-bifurcations.

\section{Dynamic factors for slow relaxations}

Let us start with some simple considerations and three explicitly integrated examples. We can immediately give two simple mechanisms for the generation of slow relaxations. It is a delay of the motion near the unstable fixed point and (for the systems that are dependent of the parameter) a delay of the motion in the region, where at small variations of the parameter there appears a fixed point.

The delay near the unstable fixed point is observed in the system $\dot{x}=x^{2}-1$. We will consider it over the segment $[-1,1]$. Here, there are two 
fixed points $x= \pm 1$. The point $x=-1$ is stable, whereas the point $x=1$ is unstable. The equation is integrated in the explicit form

$$
\begin{aligned}
x(t) & =\frac{1-A \mathrm{e}^{2 t}}{1+A \mathrm{e}^{2 t}} \\
A & =\frac{1-x_{0}}{1+x_{0}}
\end{aligned}
$$

where $x_{0}=x(0)$ is the $x$ value at the initial moment. If $x_{0} \neq 1$ then after some time the motion will enter the $\varepsilon$ neighbourhood of the point $x=-1$ (for any $\varepsilon>0$ ). This will happen after the time

$$
\begin{aligned}
\tau_{2}\left(x_{0}, \varepsilon\right) & =\tau_{3}\left(x_{0}, \varepsilon\right) \\
& =\eta_{3}\left(x_{0}, \varepsilon\right) \\
& =-\frac{1}{2} \ln \frac{\varepsilon}{2-\varepsilon}-\frac{1}{2} \ln \frac{1-x_{0}}{1+x_{0}}
\end{aligned}
$$

Naturally, it is assumed that $x_{0}>-1+\varepsilon$. Let $\varepsilon$ be fixed and $x_{0} \rightarrow 1$. Then $\tau_{2}$ and $\eta_{3}$ tend to infinity as $-(1 / 2) \ln \left(1-x_{0}\right)$.

Motion delay in the region where, for small variations of the parameter, a fixed point arises, is observed in the system $\dot{x}=\left(k+x^{2}\right)\left(x^{2}-1\right)$ (which depends on the parameter $k$ ). If $k>0$, then again there are only two fixed points, at $x= \pm 1$. For $k=0$ a third point appears at $x=0$. For $k<0$ it decomposes into two points: $x= \pm(|k|)^{1 / 2}$. Let us consider the case when $k>0$. The time of the motion form $x_{0}$ to $x_{1}$ can be found in the explicit form $\left(x_{0}>x_{1} ; x_{0}, x_{1} \neq \pm 1\right)$

$$
t\left(x_{0}, x_{1}\right)=\frac{1}{2} \ln \frac{1-x_{1}}{1+x_{1}}-\frac{1}{2} \ln \frac{1-x_{0}}{1+x_{0}}-\frac{1}{k^{1 / 2}}\left(\arctan \frac{x_{1}}{k}-\arctan \frac{x_{0}}{k}\right)
$$

In the case $\varepsilon>0, x_{0} \in(-1+\varepsilon, 1-\varepsilon)$, the relaxation times for a given system are determined as $\tau_{i}\left(x_{0}, k, \varepsilon\right)=\eta_{i}\left(x_{0}, k, \varepsilon\right)=t\left(x_{0},-1+\varepsilon\right), i=1,2$, 3. If $x>0$, then for $k \rightarrow 0$ we will have $\arctan \left(x / k^{1 / 2}\right) \rightarrow-\pi / 2$. Hence if $x_{0}>0$, $\varepsilon<1$, then for $k \rightarrow 0$, every $\tau_{i}, \eta_{i} \rightarrow \infty$ as $\pi / k^{1 / 2}$.

Slow relaxations are connected with bifurcations (explosions) of the $\omega$-limit sets since they can be caused by the delay near a "foreign" $\omega$-limit set. A "foreign" set here means the set corresponding to the motion with different initial conditions or different (but close) values of the parameters. The two simple examples above can illustrate these possibilities. The general case is described by the following theorems.

Theorem 1. $\tau_{1}$ slow relaxations exist in $\vec{X} \times \vec{K}$ if and only if $\Omega(\vec{x}, \vec{k})$-bifurcations exist in $\bar{X} \times \vec{K}$.

Theorem 2. $\eta_{1}$ slow relaxations exist in $\vec{X} \times \vec{K}$ if and only if $\Omega(\vec{k})$-bifurcations exist in $\bar{K}$.

Relaxation times $\tau_{1}$ and $\eta_{1}$ are the "shortest" in the system of times $\tau_{i}, \eta_{i}$. 
That is why their existence requires the "strongest" $\Omega$-bifurcations. For relaxation times $\tau_{2,3}$ and $\eta_{2,3}$ there exist no one-to-one relations between slow relaxations and bifurcations such as in Theorems 1 and 2. For the existence of $\tau_{2}$ and $\eta_{2}$ slow relaxations, it suffices that weaker bifurcations take place.

Theorem 3. If the system has $\omega(\vec{x}, \vec{k})$-bifurcations, $\tau_{2}$ slow relaxations also exist.

Theorem 4. If the system has $\omega_{T}(\vec{k})$-bifurcations, then there are also $\eta_{2}$ slow relaxations.

There are examples when $\tau_{2}, \eta_{2}$ slow relaxations take place without bifurcations. So far, complete characteristics of these slow relaxations in terms of the limit behaviour for a dynamic system (that is dependent on the parameters) has not been obtained. Only some of the additional sufficient conditions have been defined.

Theorem 5. If, in $\vec{X}$ for some $\vec{k} \in \vec{K}$ there exists a whole $(\vec{x}, \vec{k})$-motion for which $\alpha(\vec{x}, \vec{k}) \not \omega(\vec{x}, \vec{k})$, then there exist $\tau_{2}$ slow relaxations.

Theorem 6 . If, in $\vec{X}$ for some $\vec{k} \in \bar{K}$ there exists a whole $(\vec{x}, \vec{k})$-motion for which $\alpha(\vec{x}, \vec{k})$ does not lie entirely in the closure of $\omega_{T}(\vec{k})\left[\alpha(\vec{x}, \vec{k}) \not f \omega_{T}(\vec{k})\right]$, then there exist $\eta_{2}$ slow relaxations.

It is possible to give examples (in $R^{3}$ ) which show that the conditions of Theorems 5 and 6 can be satisfied in the absence of bifurcations.

For $\tau_{3}, \eta_{3}$ slow relaxations, the necessary and sufficient conditions have been obtained in terms of the limit behaviour of dynamic systems. Note that the $(\vec{x}, \vec{k})$-motion is called positively Poisson-stable ( $\mathrm{P}^{+}$-stable) if $\left.\vec{x} \in \omega \vec{x}, k\right)$.

Theorem 7. $\tau_{3}$ slow relaxations in $\vec{X} \times \vec{K}$ exist if and only if at least one of the following conditions is fulfilled.

(1) There exist $\omega(\vec{x}, \vec{k})$-bifurcations in $\vec{X} \times \vec{K}$.

(2) For some $\vec{k} \in \vec{K}$ in $\dot{X}$ there exists a whole $(\vec{x}, \vec{k})$-motion which is not positively Poisson-stable, and $\alpha(\vec{x}, \vec{k}) \bigcap \omega(\vec{x}, \vec{k}) \neq \phi$.

Theorem 8. $\eta_{3}$ slow relaxations in $\vec{X} \times \vec{K}$ exist if and only if at least one of the following conditions is satisfied.

(1) There exist $\omega(\vec{k})$-bifurcations in $\vec{K}$.

(2) For some $\vec{k} \in \vec{K}$ in $\bar{X}$ there exists a whole $(\vec{x}, \vec{k})$-motion not intersecting the closure of $\omega_{T}(\vec{k})$.

In theorem 7, condition (2) is satisfied by any point of the loop (a loop is the trajectory starting from some fixed point and returning to the same point). In theorem 8 , condition (2) is satisfied by any point of the trajectory from some unstable to a stable fixed point (if the points of this trajectory are not $\omega$-limit points).

At the end of this section, we will consider individual systems (without parameters) and will not point this out especially.

Let us recall the concept of the non-wandering point. It is such a point $\vec{x} \in \bar{X}$ that for any $t_{0}>0$ (arbitrarily high) and $\varepsilon>0$ (arbitrarily low) there exist such $t>t_{0}$ and $\vec{y} \in \bar{X}$ that

$$
\rho(\vec{y}, \vec{x})<\varepsilon \text { and } \rho\left(\vec{T}_{t}(\vec{y}), \vec{x}\right)<\varepsilon
$$


Theorem 9. Let some system have $\eta_{2}$ slow relaxations. Then we can find a non-wandering point $\vec{x}{ }^{*} \in \vec{X}$ that does not lie in $\overline{\omega_{T}}$.

This necessary condition for $\eta_{2}$ slow relaxations is not sufficient, which can be illustrated by the corresponding examples.

Theorem 10. Let $\dot{X}$ be connected and $\overline{\omega_{T}}$ be disconnected. Then the system will have $\eta_{3}$ and $\tau_{1,2,3}$ slow relaxations.

In accordance with theorem 9 and the Pugh lemma [22], an individual $\mathrm{C}^{1}$-smooth system "almost never" has $\eta_{2}$, slow relaxations.

In the two-dimensional case (two variables) "almost any" $\mathrm{C}^{1}$-smooth dynamic system is rough (i.e. at small bifurcations its phase pattern deforms only slightly without qualitative variations). For rough two-dimensional systems, the $\omega$-limit set of every motion is either a fixed point or a limit cycle. The stability of these points and cycles can be checked even by a linear approximation. Mutual relationships between six different types of slow relaxations for rough two-dimensional systems are sharply simplified.

Theorem 11 . Let $\vec{M}$ be a smooth compact two-dimensional variety, $\overrightarrow{\mathrm{F}}$ a $\overrightarrow{\mathrm{C}}^{1}$-smooth rough dynamic system on $\bar{M}, \vec{X}$ a connected positively invariant subset of $\vec{M}$ (at positive times the motion remains inside $\vec{X}$ ) and $\left.\vec{F}\right|_{\vec{X}}$ the $\vec{F}$ restriction on $\vec{X}$. Then

(1) The fact that $\vec{F}_{\vec{X}}$ has $\tau_{3}$ slow relaxations means that $\tau_{1,2}$ and $\eta_{3}$ slow relaxations exist.

(2) $\left.\vec{F}\right|_{\vec{X}}$ has no $\tau_{3}$ slow relaxations if and only if $\vec{X}$ has one fixed point (and no limit cycles) or one limit cycle (and no fixed points).

(3) $\eta_{1,2}$ slow relaxations of $\left.\vec{F}\right\rceil_{\vec{x}}$ are impossible.

From a practical viewpoint it is insufficient to claim that slow relaxations do exist in a given system. It would be of interest to determine their probability. As a rule, one can naturally introduce some measure (volume, area) into the phase space of the systems under research. Let us denote it as mes. The probability of slow relaxations can be treated as a behaviour at high $t$ of the functions

$$
\begin{aligned}
& \mu_{i}(t, \varepsilon)=\operatorname{mes}\left\{\vec{x} \in \vec{X} / \tau_{i}(\vec{x}, \varepsilon)>t\right\} \\
& \theta_{i}(t, \varepsilon)=\operatorname{mes}\left\{\vec{x} \in \vec{X} / \eta_{i}(\vec{x}, \varepsilon)>t\right\}
\end{aligned}
$$

We assume that $\varepsilon$ is fixed and sufficiently small. The values of $\mu_{i}$ and $\theta_{i}$ show a measure (volume, area) for those initial conditions under which the relaxation time is greater than $t$.

It is convenient to formulate the problem so that its solution would not change with smooth changes of variables. For example, let us determine the limits for $\ln \mu_{i}(t, \varepsilon) / t$ and $\ln \theta_{i}(t, \varepsilon) / t$ at $t \rightarrow \infty$ (i.e the Lyapunov indices for these functions). For smooth rough two-dimensional systems, if $\varepsilon$ is suffciently small we will obtain

$$
\lim _{t \rightarrow \infty} \frac{\ln \mu_{1,2,3}(t, \varepsilon)}{t}=\lim _{t \rightarrow \infty} \frac{\ln \theta_{3}(t, \varepsilon)}{t}=-\min \left(\kappa_{1}, \ldots, \kappa_{n}\right)
$$


At sufficiently high $t$, the functions $\theta_{1,2}$ converge to zero. Here $n$ is the number of unstable limit motions (points and cycles) in $\vec{X}$ and $\kappa_{i}$ are determined as follows. Let $\vec{B}_{1}, \ldots, \vec{B}_{n}$ be unstable limit motions lying inside $\vec{X}$.

(1) Let $\vec{B}_{i}$ be an unstable node or a focus. Then $\kappa_{i}$ will be a trace of the matrix for the linear approximation at this point.

(2) Let $\vec{B}_{i}$ be an unstable saddle. Then $\kappa_{i}$ will be a positive eigenvalue of the matrix for the linear approximation at this point.

(3) Let $\vec{B}_{i}$ be an unstable limit cycle. Then $\kappa_{i}$ is a characteristic index of this cycle (see ref. 23, p.111).

This result can be generalized for multi-dimensional systems in which a limit set for every motion is a fixed point or a limit cycle, linear approximation matrices at fixed points have no eigenvalues in the imaginary axis and limit cycles have no multiplicators on the unit circle. In this case, $\kappa_{i}$ should be treated for fixed points as the sums of those eigenvalues that have positive real parts (they are "unstable"), and for limit cycles as the sums of unstable characteristics indices.

Note that the asymptotes of eqn. (18) include characteristics of those unstable regimes near which the motion is delayed. On the other hand, in terms of the "linear" approach to relaxation studies one must examine the characteristics of that steady-state regime towards which the motion converges. These two cases differ significantly.

\section{Taking into account small perturbations and errors of models}

Any real system is known to suffer constantly from the perturbing effects of its environment. One can hardly build a model accounting for all the perturbations. Besides, as a rule, models account for the internal properties of the system only approximately. It is these two factors that are responsible for the discrepancy between real systems and theoretical models. This discrepancy is different for various objects of modern science. For example, for the objects of planetary mechanics this discrepancy can be very small. On the other hand, in chemical kinetics (particularly in heterogeneous catalysis) it cannot be negligible. Strange as it is, taking into consideration such unpredictable discrepancies between theoretical models and real systems can simplify the situation. Perturbations "smooth out" some fine details of dynamics.

A model of perturbed motion can be $\varepsilon$-motions. The time function $\phi(t)(t \geqslant 0)$ whose values belong to $\vec{X}$, is called $\varepsilon$-motion $(\varepsilon>0)$ of the system at a given $\vec{k} \in \vec{K}$ if, for any $t_{0} \geqslant 0$ and $t \in[0, \tau]$, we have

$$
\rho\left\{\phi\left(t_{0}+t\right), \bar{T}_{t}\left[\phi\left(t_{0}, \bar{k}\right)\right]\right\}<\varepsilon
$$

In other words, if for some arbitrary point $\phi\left(t_{0}\right)$ one would consider its motion to be due to the dynamic system, the discrepancy between this 
motion and $\phi\left(t_{0}+t\right)$ would be below $\varepsilon$ for $0 \leqslant t \leqslant \tau$. Here $\tau>0$ is some duration. It is not very important to know the value of $\tau$ (only the fact that it is fixed is of importance) since in what follows we will consider the case $\varepsilon \rightarrow 0$.

There are two traditional approaches to the consideration of perturbed motions. One is the study of the motion in the presence of small continuously acting perturbations [24-30]; the other is the investigation of fluctuations caused by small random perturbations [31-34]. Our results were obtained in terms of the former approach but using some ideas of the latter.

$\varepsilon$-motions have been studied previously in differential dynamics largely in connection with Anosov theorem about $\varepsilon$-trajectories and its applications [35-39].

When studying perturbed motions, every point $\vec{x}$ is juxtaposed not one $\vec{x}$-motion but a "bundle" of $\varepsilon$-motions $\phi$ starting from this point $[\phi(0)=\vec{x}]$ for a given value of the parameter $\vec{k}$. Every $\varepsilon$-motion $\phi(t)$ is associated with an $\omega$-limit set $\omega(\phi)$. It consists of those points $\vec{y} \in \bar{X}$ for which one can find such a sequence $t_{i} \rightarrow \infty$, as $\phi\left(t_{i}\right) \rightarrow y$. Every $\vec{x} \in \bar{X}$ (initial value) at a given value of the parameter $\vec{k}$ is associated with the set of $\omega^{\varepsilon}(\vec{x}, \vec{k})$. It is the combination of those $\omega(\phi)$ for which $\phi(0)=\vec{x}$ and $\phi(t)$ is the $\varepsilon$-motion (at a given $\vec{k}$ ).

It is essential to note that at $\varepsilon \rightarrow 0$ the set $\omega^{\varepsilon}(\vec{x}, \vec{k})$ does not always tend to $\omega(\vec{x}, \vec{k})$. The set $\omega^{0}(\vec{x}, \vec{k})=\bigcap_{\varepsilon>0} \omega^{6}(\vec{x}, \vec{k})$ does not obligatorily coincide with $\omega(\vec{x}, \vec{k})$ (but it is evident that it always includes the latter). In a similar way we introduce the set $\omega_{T}^{0}(\vec{k})=\bigcap_{\vec{x} \in \bar{X}} \omega^{0}(\vec{x}, \vec{k})$. It can also be wider than $\omega(\vec{k})$. The sets $\omega^{0}(\vec{x}, \vec{k})$ and $\omega^{0}(\vec{k})$ are closed and $\vec{k}$ invariant (consist of whole trajectories at a given $\vec{k}$ ). The following result seems to be essential.

Theorem 12. The functions $\omega^{0}(\vec{x}, \vec{k})$ and $\omega_{T}^{0}(\vec{k})$ are upper semicontinuous.

Note that (see ref. 40, pp. 78-83), for metric spaces, the dependence of the set on the parameter $\vec{U}(\vec{k})$ is called upper semicontinuous if, for any converging sequences $\vec{k} \rightarrow \vec{k}^{*}, \vec{y}_{i} \in \vec{U}\left(\vec{k}_{i}\right)$ and $\vec{y}_{i} \rightarrow \vec{y}^{*}$, the limit point $\vec{y}^{*}$ belongs to $\vec{U}\left(\vec{k}^{*}\right)$.

In the general case the result that is similar to Theorem 12 for ordinary limit sets $\omega(\vec{x}, \vec{k})$ and $\omega(\vec{k})$ is incorrect. It follows from this theorem that the sets of $\omega^{0}$-bifurcations are thin. The pair $\left(\vec{x}^{*}, \vec{k}^{*}\right)$ is called a point of $\omega^{0}(\vec{x}$, $\vec{k}$ )-bifurcations with the gap $\geqslant \gamma$ if there exist such sequences $\vec{x}_{i} \rightarrow \vec{x}^{*}$ and $\vec{k}_{i} \rightarrow \vec{k}^{*}$ and such a point $\vec{y} \in \omega^{0}\left(\vec{x}^{*}, \vec{k}^{*}\right)$ that for every $i$ value $\rho\left[\vec{y}, \omega^{0}\left(\vec{x}_{i}\right.\right.$, $\left.\vec{k}_{i}\right) \geqslant \gamma$, i.e. the distance from $\vec{y}$ for all the $\omega^{0}\left(\vec{x}_{i}, \vec{k}_{i}\right)$ is $\geqslant \gamma$. Similarly, the point $\bar{k}^{*}$ will be called a point of $\omega^{0}(\vec{k})$-bifurcations with a gap $\geqslant \gamma$ if there exists such a sequence $\vec{k}_{i} \rightarrow \vec{k}^{*}$ and such a point $\vec{y}=\omega^{0}(\vec{k})$ that $\rho \vec{y}$, $\left.\omega^{0}\left(\vec{k}_{i}\right)\right] \geqslant \gamma$.

Theorem 13. The set of all the points of $\omega^{0}(\vec{x}, \vec{k})$-bifurcations with the gap $\geqslant \gamma$ is nowhere dense in $\vec{X} \times \vec{K}$ for any $\gamma>0$. The set of all the points of $\omega^{0}(\vec{k})$-bifurcations with the gap $\geqslant \gamma$ is nowhere dense in $\vec{K}$ for any $\gamma>0$ either. For every $\vec{k}$ the set of each $\vec{x}$ as the $(\vec{x}, \vec{k})$ is the point of $\omega^{0}(\vec{x}$, $k$ )-bifurcations (at an arbitrary gap), is positively invariant (i.e. it consists of positive semi-trajectories). 
For every $\varepsilon$-motion of $\phi(t)$ at a given $\vec{k}$ one can introduce the following relaxation times. Let $\vec{x}=\phi(0), \gamma>0$ and

$$
\begin{aligned}
& \tau_{1}^{\varepsilon}(\phi, \vec{k}, \gamma)=\inf \left\{t \geqslant 0 / \rho\left[\phi(t), \omega^{\varepsilon}(\vec{x}, \vec{k})\right]<\gamma\right\} \\
& \tau_{2}^{\varepsilon}(\phi, \vec{k}, \gamma)=\operatorname{mes}\left\{t \geqslant 0 / \rho\left[\phi(t), \omega^{\varepsilon}(\vec{x}, \vec{k})\right] \geqslant \gamma\right\} \\
& \tau_{3}^{\varepsilon}(\phi, \vec{k}, \gamma)=\inf \left\{t \geqslant 0 / \rho\left[\phi\left(t^{\prime}\right), \omega^{\varepsilon}(\vec{x}, \vec{k})\right] \leqslant \gamma, \text { if } t^{\prime}>t\right\} \\
& \eta_{1}^{\varepsilon}(\phi, \vec{k}, \gamma)=\inf \left\{t \geqslant 0 / \rho\left[\phi(t), \omega^{\varepsilon}(\vec{k})\right]<\gamma\right\} \\
& \eta_{2}^{\varepsilon}(\phi, \vec{k}, \gamma)=\operatorname{mes}\left\{t \geqslant 0 / \rho\left[\phi(t), \omega^{\varepsilon}(\vec{k})\right] \geqslant \gamma\right\} \\
& \eta_{3}^{\varepsilon}(\phi, \vec{k}, \gamma)=\inf \left\{t \geqslant 0 / \rho\left[\phi\left(t^{\prime}\right), \omega^{\varepsilon}(\vec{k})\right] \leqslant \gamma, \text { if } t^{\prime}>t\right\}
\end{aligned}
$$

The above relaxation times can be ascribed to many various types of slow relaxation: the unrestrictedness of $\tau_{i}^{c}\left(\eta_{i}^{c}\right)$ at a given $\varepsilon$, its unrestrictedness at any sufficiently small $\varepsilon$, etc. We will consider only one case of the unrestrictedness within the limit $\varepsilon \rightarrow 0$.

Let us assume that some dynamic system has $\tau_{i}^{0}\left(\eta_{i}^{0}\right)$-slow relaxations if there exist $\gamma>0$ sequences of $\varepsilon_{j}>0, \varepsilon_{j} \rightarrow 0$ of points $\vec{x}_{j} \in \bar{X}$ and $\vec{k}_{j} \in \vec{K}$, and of $\varepsilon_{j}$-motions $\phi_{j}(t)\left(\right.$ at $\left.\vec{k}=\vec{k}_{j}\right)$ and $\phi_{j}(0)=\vec{x}_{j}$, for which at $j \rightarrow \infty$ we have

$$
\tau_{i}^{\varepsilon_{j}}\left(\phi_{j}, \vec{k}_{j}, \gamma\right) \rightarrow \infty\left[\eta_{i}^{\varepsilon_{j}}\left(\phi_{j}, \vec{k}_{j}, \gamma\right) \rightarrow \infty\right] .
$$

Theorem 14. Let $\vec{X}$ be connected. In this case $\tau_{3}^{0}$-slow relaxations exist if and only if there are $\omega^{0}(\vec{x}, \vec{k})$-bifurcations.

Let us compare this theorem with Theorem 7 about $\tau_{3}$-slow relaxations. Loops as a separate reason for slow relaxations have vanished.

Without going into details of the investigations of the relationships between slow relaxations in perturbed systems and the specificity of their dynamics, let us describe the situations in one individual system (without the parameter). In what follows the absence of the parameter is not specifically noted.

To study the limit behaviour of $\varepsilon$-motions (at $\varepsilon \rightarrow 0$ ), we will introduce two relationships [18]: pre-order $(>)$ and equivalence $(\sim)$. Let us suggest that $\vec{x}_{1}$ precedes $\vec{x}_{2}\left(\vec{x}_{1}>\vec{x}_{2}\right)$ if, for any $\varepsilon>0$, there exists such $\varepsilon$-motion $\phi$ and a moment $t>0$, as $\phi(0)=\vec{x}_{1}$ and $\phi(t)=\vec{x}_{2}$. We will claim that $\vec{x}_{1}$ is equivalent to $\vec{x}_{2}\left(\vec{x}_{1} \sim \vec{x}_{2}\right)$, if $\vec{x}_{1} \succ \vec{x}_{2}$ and $\vec{x}_{2} \succ \vec{x}_{1}$.

The relationships, rather similar in sense, for smooth dynamic systems were introduced in ref. 34 (p. 220 etc.) for studying the random perturbations via a method of action functionals. Close concepts can also be found in ref. 39.

Let us identify equivalent points in $\omega_{T}^{0}$ which is totally disconnected (each point has a system of neighbourhoods that are closed and open simultaneously). The space $\omega_{T}^{0} / \sim$ can be treated as a system of sources and sinks. This system is similar to that of limit cycles and fixed points in a smooth rough two-dimensional system. The sets $\omega^{0}(\vec{x})$ can change jumpwise only on 
the boundaries of the attraction regions for the corresponding sinks. Let us give a strict formulation of this theorem.

The set $\vec{Y} \subset \omega_{T}^{0}$ will be called saturated downwards if for any $\vec{y} \in \vec{Y}$ we have

$$
\left\{\vec{x} \in \omega_{T}^{0} \mid \vec{y} \succ \vec{x}\right\} \subset \vec{Y}
$$

It means that all the points of $\omega_{T}^{0}$ that are preceded by at least one point of $\vec{Y}$ also lie in $\vec{Y}$.

Let us determine the attraction region of the set $\grave{Y} \subset \omega_{T}^{0}$

$$
\vec{A} t^{0}(\vec{Y})=\left\{\vec{x} \in \vec{X} \mid \omega_{T}^{0}(\vec{x}) \subset \vec{Y}\right\}
$$

It consists of those $\vec{x}$ for which the limit set $\omega^{0}(\vec{x})$ lies in $\vec{Y}$. If $\vec{Y} \subset \omega_{T}^{0}$ is open in the $\omega_{T}^{0}$ saturated downwards set, then the set $\vec{A} t^{0}(\vec{Y})$ is open in $X$.

Theorem 15. Let $\vec{x}^{*}$ be the point of $\omega^{0}(\vec{x})$-bifurcations ( $\vec{k}$ has been omitted, the system has no parameter). Then there exists an open in $\omega_{T}^{0}$ saturated downwards set $\vec{Y}$ for which $\vec{x}^{*} \in \partial \vec{A} t^{0}(\vec{Y})$ (the boundary $\partial \vec{U}$ for the set $\vec{U}$ is determined as a difference of its closure $\vec{U}$ and the interior int $\vec{U}$ : $\partial \vec{U}=\overrightarrow{\bar{U}} \operatorname{int} \vec{U}$, for the open set $\partial \vec{U}=\overrightarrow{\bar{U}} \vec{U}$ ).

The relationship between $\tau_{i}^{0}$-and $\eta_{i}^{0}$-slow relaxations in individual systems is similar to that between $\tau_{i}$ - and $\eta_{i}$-slow relaxations in a smooth rough two-dimensional system (see Theorem 11).

Theorem 16. Let $\vec{X}$ be connected. Then if $\omega_{T}^{0}$ is connected, there are no $\tau_{i}^{0}$ and $\eta_{i}^{0}$-slow relaxations. In case $\omega_{T}^{0}$ is disconnected there will be $\tau_{1,2,3}^{0}$ and $\eta_{3}^{0}$-slow relaxations. $\eta_{1,2}^{0}$-slow relaxations in individual system (without parameters) are impossible.

"Structurally stable systems are not dense": this is the title of Smale's study [41] that has opened a new period in understanding dynamics. Structurally stable (rough) systems are those whose phase patterns undergo no qualitative changes at small perturbations (for accurate definitions with comprehensive motivation, see ref. 11). Smale constructed such a structurally unstable system that any system sufficiently close to it is also structurally unstable. This result has destroyed any hope of the possibility of classifying "almost all" dynamic systems. Such hopes were associated with the advance in the classification of two-dimensional systems, among which the structurally stable ones are dense.

The literature reports various attempts to improve the catastrophic situation with structural stability. One approach is to suggest such a natural concept of stability for which almost all the systems would be stable. An interesting attempt has been made in this direction [42, 43]. The following concept of stability was suggested $[42,43]$ : those systems are stable in which almost all trajectories change only slightly with small perturbations. This stability is typical. In this sense almost all the systems are stable.

The other attempt to get rid of the "Smale nightmare" (the presence of domains of structurally unstable systems) was to consider the $\varepsilon$-motion (with or without further examination of the limit $\varepsilon \rightarrow 0$ ). The picture obtained is 
more stable than the phase pattern. It seems to be evident that primary investigation must be carried out for those (more rough) details of dynamics that do not vanish at small perturbations. This approach to study transient processes was suggested by Gorban' [18]. One must note its ideological closeness to the method of quasi-mean values in statistical physics (see ref. 44, pp. 193-269). By analogy, the approach suggested can be called a method of "quasi-limit" sets.

\section{Conclusion}

Let us present the most important results of our investigations.

(1) It is not always necessary to search for some "side" reasons for slow relaxations. One must first determine whether the system has slow relaxations of dynamic (in chemistry of kinetic) origin.

(2) One of the possible reasons for slow relaxations are bifurcations (explosions) of $\omega$-limit sets. It is useful to study the dependence of $\omega \vec{x}$, $\vec{k}$ )-limit sets on the parameters and initial data. (As usual, we study the dependences only on the parameters.)

(3) Perturbations rough the system. Relationships between various types of slow relaxations taking into account perturbations are similar to those in smooth rough two-dimensional systems.

(4) Due to the large number of various types of slow relaxations irreducible to each other, in treating experimental data one must establish precisely what relaxation time is high.

(5) Slow relaxations in real systems are often "restrictedly slow", i.e. relaxation time is high but still restricted. To examine these peculiarities we recommend the following approach, originating in its significance from Andronov's study. The system considered is included in the appropriate class, for which slow (infinitely) relaxations and bifurcations are studied. This study and the information concerning the extent to which some special systems are close to the original one provide essential data. (In terms of this approach we have made an attempt to study the effect of "non-physical", in particular negative, steady states on the relaxation of catalytic reactions $[16,45]$.)

\section{References}

1 S.P. Levchenko, N.V. Kul'kova and M.I. Temkin, Kinet. Katal., 17 (1976) 1542.

2 M.I. Temkin, Kinet. Katal., 17 (1976) 1095.

3 G.D. Birkhoff, Dynamical Systems, American Mathematical Society, New York, 1927; Gostekhizdat, Moscow, 1940 (in Russian).

4 V.V. Nemytskii and V.V. Stepanov, Qualitative Theory of Differential Equations, Gos. Izd. Teor. Tekh. Lit., Moscow, Leningrad, 1949 (in Russian); Princeton University Press, Princeton, 1960. 
5 W.H. Gottschalk and G.A. Hedlund, American Mathematical Society Colloquium, Vol. 36, 1955 , p. 1.

6 W. H. Gottschalk, Bibliography for Topological Dynamics, Wesleyan University, Middletown, CT, 1966.

7 K.S. Sibirskii, Introduction to Topological Dynamics, RIO Akad. Nauk Moldavian SSR, Kishinev, 1970 (in Russian).

8 V.I. Zubov, Stability of Motion, Vysshaya Shkola, Moskva, 1973 (in Russian).

9 S. Smale, Bull. Am. Math. Soc., 73 (1967) 747; Usp. Mat. Nauk, 25 (1970) 113.

10 Z. Nitecki, Differentiable Dynamics. An Introduction to the Orbit Structure of Diffeomorphisms, MIT Press, Cambridge, MA, 1971; Mir, Moscow, 1975 (in Russian).

11 V.I. Arnold, Supplementary Chapters to Ordinary Differential Equations Theory, Nauka, Moscow, 1978 (in Russian).

12 R. Bowen, Symbolic Dynamics (Collection of Works), Mir, Moscow, 1979 (in Russian).

13 A.N. Gorban', V.I. Elokhin, V.M. Cheresiz and G.S. Yablonskii, Instationary Processes in Catalysis, Institute of Catalysis, Novosibirsk, 1979, Part 1, pp. 83-88 (in Russian).

14 A.N. Gorban', V.M. Cheresiz, V.I. Elokhin and G.S. Yablonskii, Mathematical Methods in Chemistry, Vol. 2, Qualitative Methods, CNIITENeftekhim, Moscow, 1980, pp. 53-60 (in Russian).

15 V.I. Elokhin, V.M. Cheresiz, G.S. Yablonskii and A.N. Gorban', Proc. 3rd All-Union Conf. on Kinetics of Catalytic Reactions (Kinetics-3), Vol. 1, Kalinin, 1980, pp. 152-158 (in Russian).

16 V.I. Elokhin, G.S. Yablonskii, A.N. Gorban' and V.M. Cheresiz, React. Kinet. Catal. Lett., $15(1980) 245$.

17 A.N. Gorban' and V.M. Cheresiz, Slow Relaxations of Dynamical Systems and Bifurcations of $\omega$-Limit Sets, Prepr., Computer Centre, Krasnoyarsk, 1980 (in Russian).

18 A.N. Gorban', Slow Relaxations of Perturbed Systems, Prepr. No. 27, Computer Centre, Krasnoyarsk, 1980 (in Russian).

19 A.N. Gorban' and V.M. Cheresiz, Dokl. Akad. Nauk SSSR, 261 (1981) 1050; Sov. Math. Dokl., 24 (1981) 645.

20 H.F. De Baggis, Dynamic Systems with Stable Structures. Contributions to the Theory of Non-Linear Oscillations, Vol. 2, Princeton University Press, Princeton, 1952, p. 306. Usp. Mat. Nauk, 10 (1955) 101.

21 M. Peixoto, Topology, 1 (1962) 101.

22 Ch. Pugh, Am. J. Math., 89 (1967) 956; Matematika, 12 (1968) 81, 136.

23 N.N. Bautin and E.A. Leontovich, Qualitative Investigation Methods for Dynamic Systems on the Plane, Nauka, Moscow, 1976 (in Russian).

24 G.N. Duboshin, Tr. Astronom. Inst. P.K. Sternberga, 14 (1940) 153.

25 I.G. Malkin, Prikl. Mat. Mekh., 8 (1944) 241.

26 V.E. Germaidze and N.N. Krasovskii, Prikl. Mat. Mekh., 21 ((1957) 769.

27 N.N. Krasorskii, Some Problems of Motion Stability, Fizmatgiz, Moscow, 1959 (in Russian).

28 I.G. Malkin, Theory of Motion Stability, Nauka, Moscow, 1966 (in Russian).

29 A. Strauss and A. Yorke, Bull. Am. Math. Soc., 22 (1969) 513.

30 A.A. Martynyuk and R. Gutovski, Integral Inequalities and Motion Stability, Naukova Dumka, Kiev, 1979, pp. 139-179 (in Russian).

31 L.S. Pontryagin, A.A. Andronov and A.A. Vitt, Zh. Eksp. Teor. Fiz., 3 (1933) 165.

32 A.D. Ventzel and M.I. Freidlin, Usp. Mat. Nauk, 25 (1970) 3.

33 Yu.I. Kifer, Izv. Akad. Nauk SSSR Mat., 38 (1974) 1091.

34 A.D. Ventzel and M.I. Freidlin, Fluctuations in Dynamic Systems Caused by Small Random Perturbations, Nauka, Moscow, 1979 (in Russian).

35 D.V. Anosov, Proc. Int. Conf. on Nonlinear Oscillations, Kiev, 1970, Vol. 2, pp. 39-45.

36 P. Walters, Lect. Notes Math., 668 (1978) 231.

37 J.E. Franke and J.F. Selgrade, J. Diff. Eqn., 26 (1977) 27.

38 J.E. Franke and J.F. Selgrade, Trans. Am. Math. Soc., 245 (1978) 251. 
$39 \quad$ H. Easton, Lect. Notes Math., 668 (1978) 95.

40 K. Kuratowski, Topology, Vols. 1, 2, Mir, Moscow, 1969 (in Russian).

41 S. Smale, Am. J. Math., 88 (1966) 491; Matematika, 11 (1967) 107.

42 V.A. Dobrynskii and A.N. Sharkovskii, Dokl. Akad. Nauk SSSR, 211 (1973) 273.

43 A.N. Sharkovskii, Abh. Akad. Wiss. D.D.R. Abt. Math. Naturwiss. Tech., 4 (1977) 193.

44 N.N. Bogolyubov, Selected Works on Statistical Physics, Moscow University, Moscow, 1979 (in Russian).

45 A.N. Gorban', V.I. Bykov and G.S. Yablonskii, Sketches on Chemical Relaxation, Nauka, Novosibirsk, 1986 (in Russian). 